\title{
Relação entre a avaliação de desempenho da atenção básica e a mortalidade infantil no Brasil
}

\author{
The relationship between the assessment of performance \\ of primary health care and infant mortality in Brazil
}

Marla Fabiula de Barros Hatisuka (https://orcid.org/0000-0002-9860-0498) 1,2

Ricardo Castanho Moreira (https://orcid.org/0000-0003-4014-3201) ${ }^{2}$

Marcos Aparecido Sarria Cabrera (https://orcid.org/0000-0001-9786-9674) ${ }^{1}$
${ }^{1}$ Universidade Estadual de Londrina. Rodovia Celso Garcia Cid, PR-445, Km 380, Campus Universitário. 86057-970 Londrina PR Brasil.marla@uenp.edu.br ${ }^{2}$ Universidade Estadual do Norte do Paraná. Bandeirantes PR Brasil.

\begin{abstract}
The article assesses the relationship between the Infant Mortality Rate (IMR) and the percentage of health units that obtained good performance ratings in the appraisal by the National Program to Improve Access and Quality (PMAQ) of Primary Care in Brazilian states and the relation to the variables involved. Using a descriptive study, the results of the performance assessment of the participating units of the third cycle of PMAQ (2015-2017) and the relationship with the IMR (2015-2017) were analyzed. Descriptive, Pearson correlation, and cluster analysis were performed. The results revealed that there is a negative and moderate correlation (-0.534) between the IMR and the units with a good and statistically significant ( $p=0.005$ ) performance rating. With cluster analysis, it was possible to confirm that Brazilian states from different regions reveal similarities in the variables of the study. Thus, there is a relationship between IMR and units with a good performance rating. The results also showed that the similarities between the states are not restricted to the region in which they are located. Therefore, the importance of investing in primary care training is paramount in terms of an effective impact on the health of the population.

Key words Primary Health Care, Assessment of Health Programs and Projects, Assessment of the Impact on Health, Infant Mortality
\end{abstract}

Resumo $O$ artigo verifica se há relação entre a Taxa de Mortalidade Infantil (TMI) e o percentual de unidades de saúde que obtiveram boa classificação de desempenho na avaliação do Programa Nacional de Melhoria do Acesso e da Qualidade da atenção básica (PMAQ) nos estados brasileiros, e a similaridade entre estes estados em relação a essas variáveis. Utilizando-se de um estudo descritivo, foram analisados os resultados da avaliação de desempenho das unidades participantes do $3^{\circ}$ ciclo do PMAQ (2015-2017) e a relação com a TMI (2015-2017). Foram realizadas análises descritivas, de correlação de Pearson e análise de cluster. Os resultados demonstraram que há correlação negativa e moderada $(-0,534)$ entre a TMI e as unidades com boa classificação de desempenho e estatisticamente significante $(p=0,005)$. Com a análise de cluster, foi possível verificar que estados de diferentes regiões apresentam similaridade nas variáveis do estudo. Enfim, existe relação entre a TMI às unidades com boa classificação de desempenho, além disso, verificou-se que a similaridade entre os estados vai além da região a que pertencem. Portanto, fica evidente a importância do investimento na qualificação da AB para o impacto efetivo na saúde da população.

Palavras-chave Atenção Primária à Saúde, Avaliação de Programas e Projetos de Saúde, Avaliação do Impacto na Saúde, Mortalidade Infantil 


\section{Introdução}

Os indicadores de saúde, em especial o de Mortalidade Infantil (MI), são considerados importantes fontes de informação tanto a respeito das características do estado de saúde da população, quanto do sistema de saúde ao qual a população tem acesso, deste modo, quando vistos em conjunto, servem para o acompanhamento e planejamento das ações em saúde pública ${ }^{1}$.

Assim, com o cálculo da Taxa de Mortalidade Infantil (TMI) estima-se a possibilidade de óbito em crianças menores de um ano, numa determinada região e num período específico, podendo nortear ações para a diminuição destes óbitos, tanto na área da saúde, como nos demais setores ${ }^{1}$.

Desta maneira, a TMI é um indicador fortemente influenciado pelos determinantes sociais, como inclusão social, segurança, moradia, renda, escolaridade, estilo de vida, modelo de atenção à saúde e acesso aos serviços de saúde, entre outros $^{2}$.

Nas últimas décadas o Brasil reduziu significativamente as $\mathrm{TMI}^{3}$, dentre as ações que resultaram nesta diminuição, tem-se à ampliação dos serviços de saúde, através da expansão da Atenção Básica $(\mathrm{AB})$, tendo como principal representante a Estratégia Saúde da Família (ESF) ${ }^{4}$. Este modelo de atenção foi definido pelo Ministério da Saúde (MS) como coordenador da assistência à Saúde, com a finalidade de subsidiar a integralidade das ações e dos serviços de saúde disponíveis à população. Assim, as ações para a redução da TMI devem ser auxiliadas por meio do conhecimento das condições de vida e da saúde da população adstrita à unidade de saúde $e^{4}$.

Deste modo, a intensa expansão da ESF, desde sua implantação na década de 1990, e consequentemente o aumento da cobertura da população por este modelo de atenção, vários esforços foram realizados para a qualificação dos profissionais que compõe este serviço, a fim de garantir que a população tenha acesso de qualidade dos serviços prestados ${ }^{5}$.

Neste sentido, no ano de 2011 o MS institui o Programa Nacional de Melhoria do Acesso e da Qualidade da Atenção Básica (PMAQ - AB) que tem como objetivo a "ampliação do acesso e a melhoria da qualidade deste nível de atenção, garantindo um padrão de qualidade comparável nacional, regional ou localmente"6.

Desde que foi instituído, este programa teve três ciclos de avaliação, considerando a infraestrutura da unidade, o alcance dos indicadores pactuados no momento da adesão, além de entrevistas com os gestores, os profissionais de saúde e os usuários dos serviços, assim ao final de cada ciclo as unidades foram classificadas de acordo com seu desempenho ${ }^{6}$.

Desta maneira, os dados gerados pelo PMAQ se tornaram uma importante fonte de dados para realização de pesquisas na área da saúde, por considerar os diversos atores deste serviço, por ser um programa de alcance nacional e pela magnitude de variáveis analisadas.

A vista disto, em pesquisa recente que utilizou os dados do $2^{\circ}$ ciclo do PMAQ, identificou ações inadequadas para o aprimoramento do pré-natal, além de relacionar as desigualdades regionais ao acesso deste serviço em todo país. Este é um importante achado para direcionar as ações da $\mathrm{AB}$, pois a qualidade do acesso, da assistência e o número de consultas de pré-natal estão diretamente relacionados à $\mathrm{TMI}^{8-10}$.

Nesta perspectiva, a avaliação de desempenho das unidades que aderiram ao PMAQ e a comparação com dados sobre as estatísticas vitais, como a TMI, são imperativas já que a proposta do programa é, entre outras, a redução das desigualdades no acesso à $\mathrm{AB}$ por meio da reorganização do modelo de atenção, utilizando a ESF ${ }^{11}$.

Considerando que o PMAQ, como política indutora de qualidade concluiu o $3^{\circ}$ ciclo de avaliação, e o impacto da $\mathrm{AB}$ na saúde da população, a presente pesquisa teve como objetivo verificar se há relação entre a TMI e o percentual de unidades de saúde que obtiveram boa classificação de desempenho na avaliação do PMAQ nos estados brasileiros, e a similaridade entre os estados em relação a essas variáveis.

\section{Método}

Trata-se de uma pesquisa exploratória, retrospectiva, de análise quantitativa, utilizando banco de dados secundários. O PMAQ foi instituído em 2011 e até o momento foram realizados três ciclos de avaliação, ao final de cada ciclo, as equipes foram classificadas de acordo com o seu desempenho, está fase também é denominada como certificação.

Para isto, as equipes foram avaliadas segundo três perspectivas, sendo: o conjunto de padrões previamente determinados (equivalente a $60 \%$ da nota de certificação); a avaliação do cumprimento dos indicadores pactuados com as unidades (30\%) e a verificação da realização no momento auto avaliativo (10\%). Desta maneira, as unidades de saúde foram classificadas pelo estabelecido no 
programa, como: ótima, muito boa, boa, regular e ruim. Algumas unidades foram classificadas como insatisfatória ou desclassificadas ${ }^{6}$.

Para o presente estudo, foram usados os dados dos resultados da classificação de desempenho das equipes de saúde dos municípios brasileiros, participantes do $3^{\circ}$ ciclo do PMAQ (2015-2017), disponíveis no portal do Departamento de Atenção Básica (DAB/MS) ${ }^{6}$. Os dados sobre a certificação foram importados e armazenados em planilhas do software Microsoft Excel 2010. Para a análise inferencial foi utilizado o pacote estatístico SPSS 20.0. Foram realizadas análises descritivas e de frequência, para isto, as avaliações da classificação de desempenho foram codificadas conforme segue: $0=$ desclassificada, $1=$ insatisfatória, $2=$ ruim, $3=$ regular, $4=$ boa, $5=$ muito boa e $6=$ ótima. Para as demais análises estatísticas, as unidades classificadas como boas, muito boas e ótimas, foram agrupadas em uma única variável renomeada como "boa classificação de desempenho".

Para efeitos desta análise, os resultados da classificação de desempenho das unidades de saúde dos municípios, foram agrupados de acordo com o Estado da Federação ao qual pertencem. Este agrupamento baseia-a se nas próprias diretrizes do $\mathrm{PMAQ}^{6}$, que propõe a construção de parâmetros que possibilitem realizar análises sobre o acesso e a qualidade dos serviços ofertados pela $\mathrm{AB}$, também de maneira regional, podendo assim desvelar possíveis desigualdades em saúde no Brasil.

Os dados para a análise da TMI foram coletados no Sistema de Informação de Mortalidade (SIM) referentes aos óbitos de menores de um ano, no período de 2015 a 2017, também foram coletados dados sobre o total de nascidos vivos, disponíveis no Sistema de Informação de Nascidos Vivos de cada estado brasileiro, pelo mesmo período. Estes sistemas são acessados através do Departamento de Informática do SUS (DATASUS), todos disponibilizados pelo Ministério da Saúde $(\mathrm{MS})^{12}$.

A TMI é representada pela razão entre o número de óbitos em menores de um ano, por local de residência, e número de total nascidos vivos, por local de residência materna, multiplicado por mil. Foram realizadas as TMI dos estados brasileiros dos anos de 2015 a $2017^{1}$.

Para verificar a associação entre as médias da TMI e as porcentagens de unidades com boa classificação de desempenho de saúde foi realizado o teste de coeficiente de correlação de Pearson, para as decisões estatísticas, foi adotado nível de significância de 5\%, sendo a TMI a variável dependente e a variável boa classificação de desempenho a independente. Para a interpretação da magnitude da correlação, adotou-se a classificação proposta por Bisquerra et al. $.^{13}:<0,20=$ muito baixa, 0,20 a $0,40=$ baixa, 0,40 a $0,60=$ moderada, 0,60 a $0,80=$ alta, e $0,80=$ muito alta, sendo a correlação de valor 1,00 considerada perfeita.

Outro aspecto relevante, para o presente estudo, foi verificar se os estados que pertencem às mesmas regiões do Brasil apresentam similaridade em relação às porcentagens de unidades com boa classificação de desempenho e nas médias da TMI. À vista disto, tem-se que a região Nordeste apresentou a expressiva expansão de ESFno Brasil $^{14}$, assim, com a análise de similaridade, pode-se verificar se este fato impactou de maneira semelhante todos os estados pertencentes a esta região.

Para isso, foi realizada a análise de cluster, verificando a dissimilaridade entre as unidades de saúde que obtiveram boa classificação de desempenho na avaliação PMAQ e a TMI de acordo com os estados brasileiros, aplicou-se o método de análise multivariada e agrupamento hierárquico (joining), adotando os seguintes procedimentos descritos por Lima e Silva ${ }^{15}$ :

1. Padronização dos dados: a padronização foi adotada para que as variáveis contribuam com o mesmo peso no cálculo do coeficiente de dissimilaridade entre eles, utilizando a seguinte equação:

$$
\mathrm{Z}=\frac{\mathrm{X}-\mu}{\mathrm{s}}
$$

Eq. 1

Em que: $Z$ = variável padronizada; $X=$ valor da variável; $\mu=$ valor médio de cada variável; e s = desvio padrão de cada variável.

2. Escolha do coeficiente de semelhança: adotou-se a medida de dissimilaridade, utilizando a distância euclidiana $\left(\mathrm{d}_{\mathrm{AB}}\right)$, pois quanto menor o valor, mais próximos estão os resultados das variáveis entre os diferentes tratamentos. A distância euclidiana $\left(\mathrm{d}_{\mathrm{AB}}\right)$ foi determinada pela equação 2 entre os acessos para o conjunto das variáveis estudadas:

$$
\mathrm{d}_{\mathrm{AB}}=\sqrt{\left(\mathrm{X}_{1 \mathrm{~A}}-\mathrm{X}_{1 \mathrm{~B}}\right)^{2}+\left(\mathrm{X}_{2 \mathrm{~A}}-\mathrm{X}_{2 \mathrm{~B}}\right)^{2}+\ldots+\left(\mathrm{X}_{\mathrm{mA}}-\mathrm{X}_{\mathrm{mB}}\right)^{2}}
$$

Em que: $\mathrm{X}_{1 \mathrm{~A}}=$ valor da variável $1^{\mathrm{a}} ; \mathrm{X}_{1 \mathrm{~B}}=$ valor da variável $1 \mathrm{~B}$.

3. Estratégia de agrupamento: foi utilizado o método ward's, que forma os grupos buscando 
minimizar a soma das diferenças entre os elementos de cada grupo e o valor médio do grupo, minimizando o desvio padrão entre os dados de cada grupo formado. O estudo foi aprovado pelo Comitê de Ética em Pesquisa da Universidade Estadual do Norte do Paraná.

\section{Resultados}

Os resultados obtidos a partir da análise da TMI e a distribuição porcentagem da classificação de desempenho estão apresentados abaixo. É interessante destacar, apenas com a análise descritiva, a relação entre as variáveis estudadas, já que o estado de Santa Catarina apresentou a maior porcentagem de unidades com boa classificação de desempenho $(69,8 \%)$ e a menor TMI $(9,54)$, enquanto o estado do Amapá apresentou a menor porcentagem de unidades com boa classificação de desempenho $(10,10 \%)$, assim também apresentou a maior TMI entre os estados brasileiros $(18,21)$ (Tabela 1).

Em relação à análise de correlação entre as variáveis do estudo, pode-se afirmar que existe correlação linear entre a TMI e a porcentagem de unidades com boa classificação de desempenho no PMAQ, indicando que quanto maior o percentual de unidades com boa classificação de desempenho, menor será a TMI, sendo esta correlação moderada $(-, 534)$ e estatisticamente significante $(\mathrm{p}=0,005)$ (Gráfico 1).

A partir da análise de cluster, obteve-se a formação de quatro grupos (Figura 1). Sendo assim, os estados pertencentes a cada grupo são similares em relação à porcentagem de unidades com boa classificação de desempenho e a TMI do estudo. Diante disto, é interessante observar os agrupamentos dos estados de diferentes regiões em cada grupo, com exceção do G2, que se formou apenas com estados da região Nordeste.

Outro ponto importante, é que o G4 ficou totalmente isolado dos demais grupos, e observando os resultados descritivos apresentados na Tabela 1, percebe-se que são os estados com as menores porcentagens de unidades com boa classificação de desempenho e maiores médias da TMI.

\section{Discussão}

Não se pretende, com este estudo, atribuir apenas aos serviços públicos de saúde a responsabilidade sobre a TMI, pois se sabe que vários são os fatores que nela interferem. Assim, as variáveis explicativas da TMI vão desde as condições sociodemográficas e socioeconômicas materna, variáveis relacionadas à assistência à saúde durante a gestação e ao parto, assim como as condições de saúde da própria criança ${ }^{16}$.

Entretanto, a $\mathrm{AB}$ tem, em seu escopo, ações voltadas para o enfrentamento, mesmo que em partes, destas variáveis e que podem influenciar nas estatísticas vitais, como o planejamento familiar, o pré-natal, a cobertura vacinal, a orientação sobre aleitamento materno, a consulta neonatal na primeira semana de vida, a puericultura e os encaminhamentos para a atenção especializada.

A vista disto, as análises aqui realizadas demonstram que há relação entre a média da TMI e a porcentagem de unidades com boa classificação de desempenho na avaliação do PMAQ e, também foi possível verificar quais os estados brasileiros que são similares em relação às médias das variáveis estudadas.

Nesta perspectiva, em estudo recente e regional, realizado no semiárido brasileiro foi constatada correlação entre a expansão da ESF e as consultas de pré-natal com a diminuição da $\mathrm{TMI}^{17}$. Embora os estados do semiárido brasileiro pertençam à mesma região, com exceção do norte de Minas Gerias ${ }^{18}$, na análise de cluster, estes ficaram em diferentes grupos, entretanto, a maioria dos estados pertenceram ao G3, logo foi possível demostrar que estes estados do Nordeste são similares aos estados das demais regiões.

Portanto, pode-se destacar que a expansão da ESF melhorou o acesso e a utilização dos serviços de saúde, principalmente da população mais vulnerável, e isso impactou na saúde da população, incluindo reduções importantes na TMI ${ }^{19}$.

Assim também, o Observatório Ibero-Americano de políticas e sistemas de saúde evidenciou o decréscimo de todos os países participantes na TMI, com destaque para o Brasil e Peru. Entretanto, os autores são incisivos ao afirmar que, apenas a expansão da cobertura da $\mathrm{AB}$ e o consumo dos cuidados deste modelo de atenção não são suficientes ${ }^{20}$.

Deste modo, além dos estímulos para a melhoria da qualidade dos serviços de saúde, são necessárias ações intersetoriais para incentivar políticas públicas que visem a diminuição das iniquidades em saúde, ao qual a população, principalmente os mais pobres, estão submetidas, como, por exemplo, aumentar o investimento em saneamento básico.

Neste sentido, foi constatado que houve aumento de óbitos por diarreia no período pós- 
Tabela 1. Distribuição das unidades de saúde segundo classificação de desempenho no PMAQ e Taxa de Mortalidade Infantil (TMI), de acordo com o estado. Brasil, 2020.

\begin{tabular}{|c|c|c|c|c|c|c|c|c|c|c|c|c|c|c|c|}
\hline \multirow{3}{*}{ Estado } & \multicolumn{14}{|c|}{ Classificação PMAQ } & \multirow{3}{*}{ TMI } \\
\hline & \multicolumn{2}{|c|}{ Desclassificado } & \multicolumn{2}{|c|}{ Insatisfatória } & \multicolumn{2}{|c|}{ Ruim } & \multicolumn{2}{|c|}{ Regular } & \multicolumn{2}{|c|}{ Boa } & \multicolumn{2}{|c|}{ Muito boa } & \multicolumn{2}{|c|}{ Ótima } & \\
\hline & $\mathbf{n}$ & $\%$ & $\mathbf{n}$ & $\%$ & $\mathbf{n}$ & $\%$ & $\mathbf{n}$ & $\%$ & $\mathbf{n}$ & $\%$ & $\mathbf{n}$ & $\%$ & $\mathbf{n}$ & $\%$ & \\
\hline $\mathrm{AC}$ & 15 & 6,8 & - & - & 86 & 38,9 & 95 & 43,0 & 20 & 9,0 & 3 & 1,4 & 2 & 0,9 & 15,33 \\
\hline $\mathrm{AL}$ & 20 & 2,1 & - & - & 116 & 12,1 & 262 & 27,3 & 325 & 33,9 & 193 & 20,1 & 43 & 4,5 & 14,12 \\
\hline AM & 42 & 5,8 & 10 & 1,4 & 227 & 31,4 & 240 & 33,2 & 147 & 20,4 & 47 & 6,5 & 9 & 1,2 & 16,02 \\
\hline AP & 11 & 5,9 & 8 & 4,3 & 83 & 44,1 & 67 & 35,6 & 8 & 4,3 & 7 & 3,7 & 4 & 2,1 & 18,21 \\
\hline BA & 152 & 4,2 & 5 & 0,1 & 404 & 11,3 & 1169 & 32,7 & 1187 & 33,2 & 523 & 14,6 & 138 & 3,9 & 15,46 \\
\hline $\mathrm{CE}$ & 296 & 11,2 & 7 & 0,3 & 201 & 7,6 & 456 & 17,2 & 755 & 28,5 & 640 & 24,1 & 298 & 11,2 & 12,63 \\
\hline ES & 42 & 5,8 & 2 & 0,3 & 119 & 16,4 & 322 & 44,4 & 189 & 26,1 & 51 & 7,0 & - & - & 11,25 \\
\hline GO & 12 & 0,8 & 3 & 0,2 & 323 & 20,9 & 689 & 44,7 & 368 & 23,9 & 116 & 7,5 & 31 & 2,0 & 12,37 \\
\hline MA & 58 & 3,1 & 5 & 0,3 & 797 & 43 & 626 & 33,8 & 274 & 14,8 & 74 & 4,0 & 20 & 1,1 & 15,34 \\
\hline MG & 256 & 4,4 & 8 & 0,1 & 611 & 10,5 & 1542 & 26,4 & 1790 & 30,6 & 1189 & 20,4 & 446 & 7,6 & 11,45 \\
\hline MS & 16 & 2,7 & 6 & 1,0 & 67 & 11,3 & 158 & 26,7 & 172 & 29,1 & 130 & 22,0 & 43 & 7,3 & 11,82 \\
\hline MT & 9 & 1,3 & 1 & 0,1 & 55 & 7,8 & 229 & 32,5 & 241 & 34,2 & 152 & 21,6 & 18 & 2,6 & 13,38 \\
\hline PA & 51 & 4,0 & 2 & 0,2 & 423 & 33,4 & 492 & 38,8 & 225 & 17,7 & 60 & 4,7 & 15 & 1,2 & 15,34 \\
\hline PB & 21 & 1,3 & 4 & 0,2 & 157 & 9,5 & 420 & 25,5 & 612 & 37,1 & 353 & 21,4 & 81 & 4,9 & 12,52 \\
\hline $\mathrm{PE}$ & 153 & 6,4 & - & - & 207 & 8,7 & 517 & 21,7 & 772 & 32,4 & 515 & 21,6 & 221 & 9,3 & 13,01 \\
\hline PI & 28 & 2,2 & - & - & 228 & 17,5 & 253 & 19,5 & 346 & 26,6 & 229 & 23,0 & 146 & 11,2 & 15,54 \\
\hline PR & 139 & 5,8 & 3 & 0,1 & 404 & 16,9 & 876 & 36,5 & 662 & 27,6 & 272 & 11,3 & 41 & 1,7 & 10,60 \\
\hline RJ & 126 & 5,0 & 6 & 0,2 & 560 & 22,4 & 570 & 22,8 & 540 & 21,6 & 630 & 25,2 & 67 & 2,7 & 12,86 \\
\hline $\mathrm{RN}$ & 2 & 0,2 & 2 & 0,2 & 137 & 11,5 & 237 & 19,9 & 345 & 28,9 & 231 & 19,4 & 239 & 20,0 & 13,01 \\
\hline RO & 65 & 17,1 & - & - & 141 & 37,1 & 86 & 22,6 & 61 & 16,1 & 25 & 6,6 & 2 & 0,5 & 13,59 \\
\hline $\mathrm{RR}$ & 2 & 1,6 & - & - & 61 & 48,0 & 26 & 20,5 & 24 & 18,9 & 14 & 11,0 & - & - & 17,70 \\
\hline RS & 38 & 2,0 & 14 & 0,7 & 189 & 9,7 & 866 & 44,6 & 551 & 28,4 & 235 & 12,1 & 47 & 2,4 & 10,12 \\
\hline SC & 27 & 1,4 & 2 & 0,1 & 119 & 6,2 & 428 & 22,5 & 665 & 34,9 & 535 & 28,1 & 130 & 6,8 & 9,54 \\
\hline SE & 51 & 7,9 & - & - & 167 & 25,7 & 154 & 23,7 & 181 & 27,9 & 84 & 12,9 & 12 & 1,8 & 15,25 \\
\hline SP & 197 & 4,0 & 27 & 0,5 & 555 & 11,2 & 1555 & 31,3 & 1664 & 33,5 & 836 & 16,8 & 135 & 2,7 & 10,93 \\
\hline TO & 17 & 2,9 & - & - & 98 & 16,5 & 220 & 37,1 & 145 & 24,5 & 92 & 15,5 & 21 & 3,5 & 12,64 \\
\hline
\end{tabular}

Fonte: Elaborado pelos autores, 2020.

neonatal nos anos de 2015 e 2016, em todas as regiões do Brasil, com exceção do Sul. Sendo este agravo considerado sensível à $\mathrm{AB}$, esses óbitos podem ser atribuídos à dificuldade de acesso aos serviços de saúde, assim como, reflete à piora das condições de vida da população $\mathrm{O}^{21,22}$. Portanto, investir na melhoria do acessoaos serviços de saúde para a população, que sofre constantes mudanças, é essencial para continuidade e melhoria dos efeitos sentidos até aqui ${ }^{19}$.

Convém mencionar que, a presente pesquisa analisou a qualidade $\mathrm{da} A \mathrm{AB}$ a partir dos resultados do PMAQ, que avaliou infraestrutura, avaliação de gestores, profissionais de saúde e usuários, além de indicadores pactuados no momento da adesão, portanto o acesso e disponibilidade dos serviços de saúde são partes integrantes desta avalição. Diante disso, deve-se manter um esforço para garantir o acesso oportuno, sem descui- dar do desenvolvimento social e de políticas que possam promover a saúde ${ }^{20}$.

Em relação à análise de cluster, observa-se que o G4 ficou completamente isolado dos demais. Pertencem a este grupo cinco estados da região Norte (Acre, Amazonas, Amapá, Pará e Roraima) e um estado da região Nordeste (Maranhão), isso demonstra a importância de avaliações para além das regiões do Brasil. Observando as análises descritivas, dispostas na Tabela 1, verifica-se que estes são os estados com a maior TMI e com menor porcentagem de unidades com boa classificação de desempenho. Apesar do aumento expressivo da cobertura da ESF nas regiões Norte e Nordeste, não, necessariamente, significam que a qualidade acompanhou este avanço.

Diante disto, temos o Maranhão, o único estado da região Nordeste que pertence ao G4 apresenta mais de $80 \%$ de cobertura da ESF, estando 


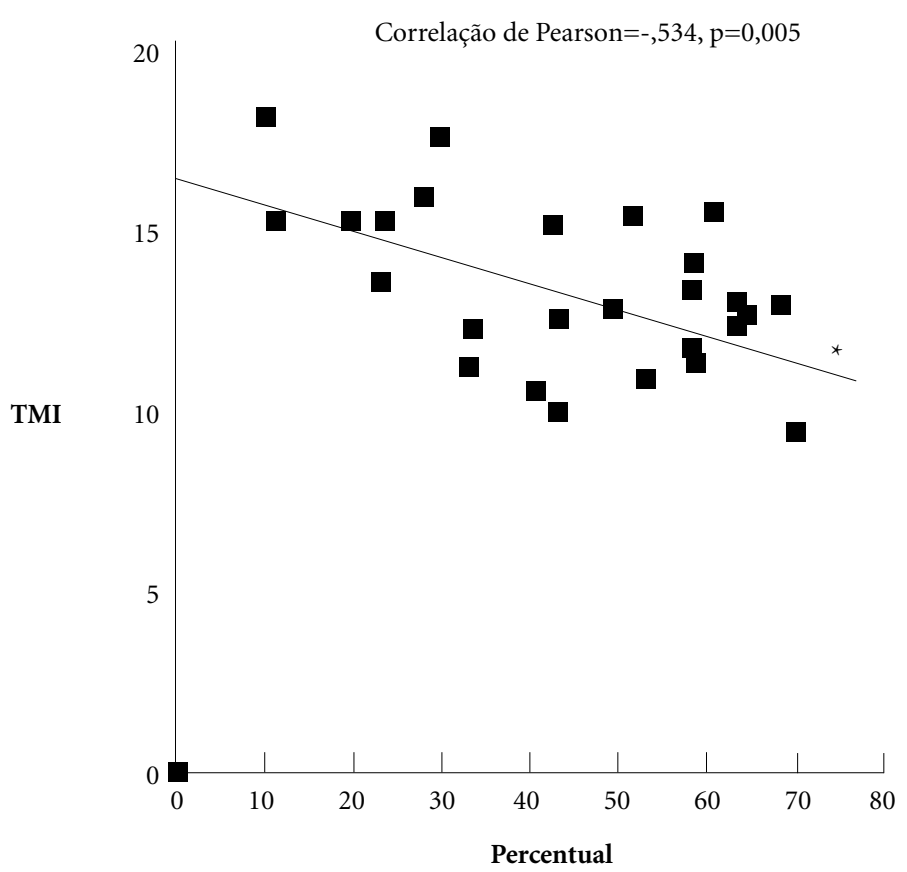

Gráfico 1. Correlação em percentual de unidades de saúde com boa classificação de desempenho e a TMI. Brasil, 2020.

Legenda: ${ }^{\star}$ Linha de Tendência.

Fonte: Elaborado pelos autores, 2020.

na média de cobertura desta região $0^{23}$, contudo, apresentou a média 19,8\% de unidades com boa avaliação de desempenho, estando muito aquém da média macrorregião que é de 54,70\%. Em relação à média da TMI deste estado também é maior do que a regional que é de 14,10/1000NV. Sabe-se que além do acesso aos serviços de qualidade, outros fatores impactam neste indicador, assim vale ressaltar que o Maranhão tem a menor renda per capita do país e um dos menores Índices de Desenvolvimento Humano do Brasil ${ }^{24}$.

Portanto, para a redução da MI é necessário que haja investimentos regionais, com foco na diminuição das desigualdades, sem abdicar dos avanços que foram alcançados até o momento. Ao contrário, fortalecendo-os com qualidade e associação aos demais setores ${ }^{25}$.

Desta maneira, evidencia-se que o efeito deste modelo de atenção à saúde da população está além da cobertura, assim iniciativas de promoção da qualificação da $\mathrm{AB}$ devem ser mantidas e aprimoradas. O pré-natal é considerado uma prática de prevenção e promoção da saúde materno-infantil, realizada pela $A B$, assim o número sufi- ciente e com qualidade desta consulta diminuem as chances de óbito fetal e infantil ${ }^{10}$. Dessa forma, o investimento em qualidade, tanto de recursos humanos como em recursos materiais devem ser permanentes para o avanço de políticas públicas com vistas a redução da TMI.

Em estudo que avaliou a qualidade do prénatal, nas macrorregiões do país, verificou que a região Norte apresentou a menor prevalência de pré-natal de qualidade ${ }^{26}$, assim também em pesquisa que utilizou dados dos primeiros ciclos do PMAQ verificou que todas as macrorregiões do país melhoraram a infraestrutura para o atendimento ao pré-natal, com exceção da região Nor$t^{23}$. Estes estudos corroboram, em parte, com a presente pesquisa, já que o G4 é composto majoritariamente por estados desta macrorregião.

Contudo, há ainda grandes desafios a serem superados para a qualificação da $\mathrm{AB}$, como a valorização das ações curativas em detrimentos às ações preventivas e as de promoção, a dificuldade em fixar os profissionais na unidade, prejudicando assim o vínculo entre profissionais e usuários, superação das dificuldades da organização do 


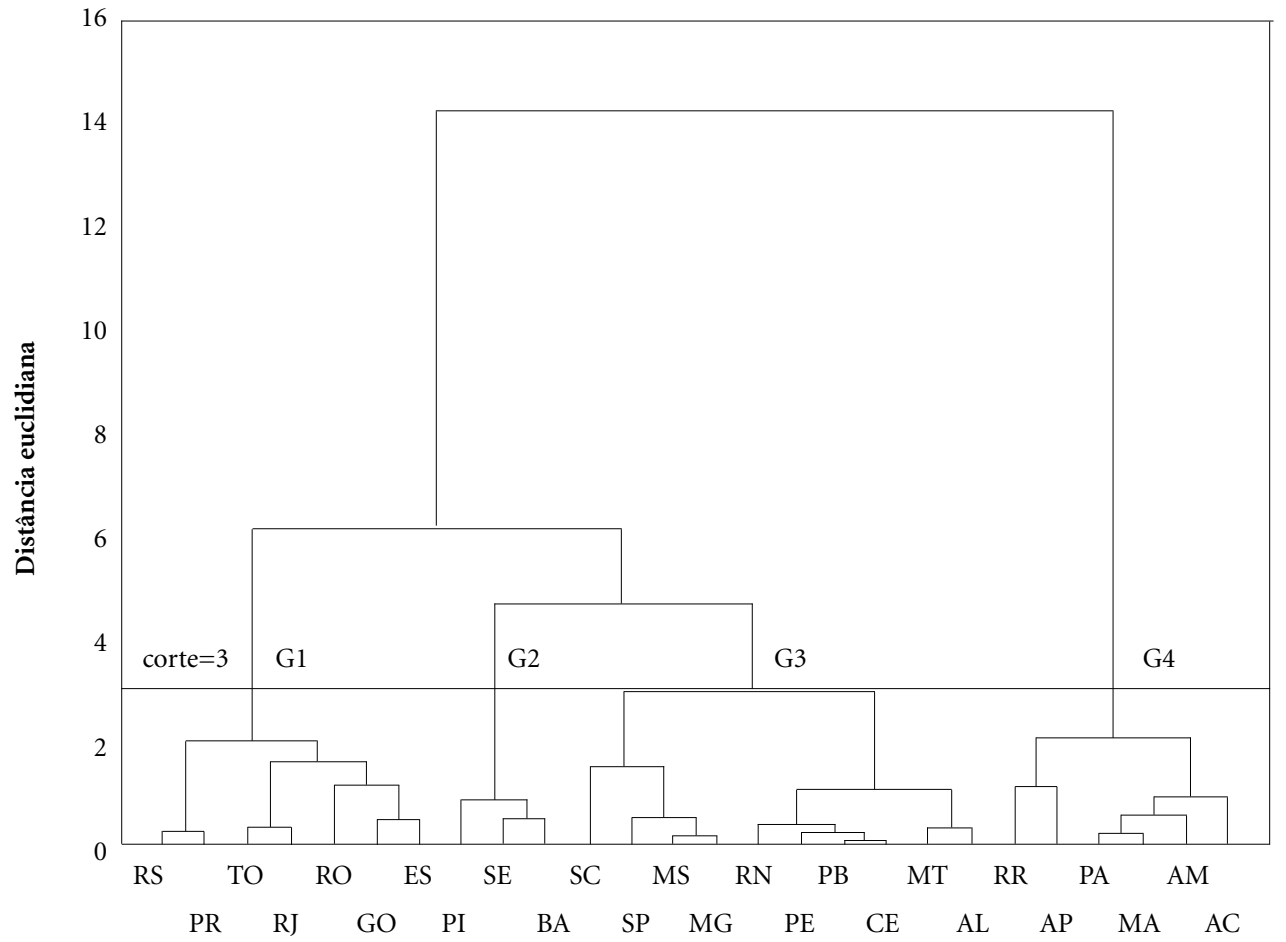

Figura 1. Dendrograma de agrupamento dos estados de acordo com o percentual de unidades com boa classificação de desempenho na avaliação PMAQ e a TMI. Brasil, 2020.

Fonte: Elaborado pelos autores, 2020.

processo de trabalho e de relacionamento interpessoal, diferentes perspectivas entre os profissionais de saúde e usuários, além das dificuldades de financiamento, como a Emenda Constitucional 95 que limita os gastos públicos em saúde, e a forma de gestão dos serviços de saúde no SUS e a sustentabilidade da $\mathrm{AB}^{27}$.

Em relação às limitações do estudo, destacase que a participação das equipes se dá de maneira voluntária, sugerindo que as unidades participantes tendem a serem as melhores e a equipe preparada para a avaliação, assim, deve-se ter cautela na generalização dos dados, pois podem estar piores do que o encontrado no presente estudo. Contudo, quando a equipe adere ao PMAQ, passa-se a refletir sobre este processo de avaliação, $\mathrm{e}$ isso deve contribuir para melhoria do acesso e da qualidade da atenção. Outro aspecto relevante é a possibilidade de vieses de informação quando se utiliza de banco de dados secundários, passíveis de erros de digitação, também podendo ocorrer subnotificação de nascimento e óbito.
Ainda como limitação do estudo, tem se a agregação dos dados e consequentemente o pequeno número de observações, entretanto, é necessário destacar o grande número de unidades participantes que o PMAQ em todo território nacional, e a comparação entre a classificação de desempenho e a TMI preenche uma lacuna existente na literatura, bem como oferece informações sobre o cenário a respeito da saúde no Brasil.

Por fim, a presente pesquisa demonstrou que existe correlação negativa entre a boa classificação de desempenho da unidade de saúde com a TMI. Também possibilitou verificar, através da análise de agrupamento a diversidades de estados nos grupos com melhores médias de TMI, com destaque para dois estados da região Norte, historicamente a macrorregião com maior TMI. Assim, o estudo demonstra a importância do investimento na qualificação da $A B$.

A avaliação de desempenho das unidades de saúde e a relação de como incidem sobre as estatísticas vitais, como a TMI, é uma importante fer- 
ramenta para os gestores, profissionais de saúde e usuários dos serviços ofertados pelo SUS. Para os gestores e profissionais da saúde, a avaliação de desempenho pode contribuir nas escolhas das estratégias e ações para garantir o acesso de qualidade dos serviços de saúde para a população. Já para os usuários, é uma maneira de obter respostas efetivas sobre os serviços públicos e sobre as políticas públicas de saúde, podendo despertar o interesse na participação social, garantida pelos fundamentos do SUS.

\section{Colaboradores}

MFB Hatisuka participou da concepção e delineamento do estudo, análise e interpretação dos resultados, redação e revisão crítica do conteúdo. RC Moreira da concepção e delineamento do estudo, análise e interpretação dos resultados. MAS Cabrera, participou da concepção e delineamento do estudo, revisão crítica do conteúdo e aprovação da versão final. 


\section{Referências}

1. Organização Pan-Americana da Saúde (OPAS). Rede Interagencial de Informação para a Saúde. Indicadores básicos para a saúde no Brasil: conceitos e aplicações. $2^{\mathrm{a}}$ ed. Brasília: OPAS; 2008.

2. Vianna LAC. Determinantes Sociais de Saúde: processo saúde doença [Internet]. [acessado 2015 jan 27]. Disponível em: https://www.unasus.unifesp.br/biblioteca_virtual/pab/7/unidades_conteudos/unidade05/ unidade05.pdf.

3. Brasil. Programa das Nações Unidas para o Desenvolvimento (PNUD). Instituto de Desenvolvimento Humano Sustentável (IDHS). Estudos temáticos sobre os objetivos de desenvolvimento do milênio da rede de laboratórios acadêmicos para acompanhamento dos objetivos de desenvolvimento do milênio: saúde: objetivo 4: reduzir a mortalidade infantil; objetivo 5; melhorar a saúde materna; objetivo 6: combate HIV/AIDS; a malária e outras doenças. Belo Horizonte: PUC Minas, UFPA, IDHS, PNUD; 2004

4. Brasil. Ministério da Saúde (MS). Portaria no 2.436, de 21 de setembro de 2017. Aprova a Política Nacional de Atenção Básica (PNAB), estabelecendo a revisão de diretrizes e normas para a organização da atenção básica, no âmbito do Sistema único de Saúde. Diário Oficial da União 2017; 22 set.

5. Medrado JRS, Casanov A, Angela O, Oliveira CCM. Estudo avaliativo do processo de trabalho das Equipes de Atenção Básica a partir do PMAQ-AB. Saude Debate 2015; 39(107):1033-1043.

6. Brasil. Ministério da Saúde (MS). Secretaria de Atenção à Saúde. Departamento de Atenção Básica. Nota Metodológica da Certificação das Equipes de Atenção Básica Programa Nacional de Melhoria do Acesso e da Qualidade da Atenção Básica (PMAQ-AB). Brasília: MS; 2018.

7. Guimarães WSG, Parente RCP, Guimarães TLF, Garnelo L. Acesso e qualidade da atenção pré-natal na Estratégia Saúde da Família: infraestrutura, cuidado e gestão. Cad Saude Publica 2018; 34(5):e00110417.

8. Kale PL, Mello-Jorge MHP, Silva KS, Fonseca KSS, Fonseca SC. Neonatal near miss and mortality: factors associated with life-threatening conditions in newborns at six public maternity hospitals in Southeast Brazil. Cad Saude Publica 2017; 33(4):e00179115.

9. Lima JC, Mingarelli AM, Segri NJ, Zavala AJZ, Takano OA. Estudo de base populacional sobre mortalidade infantil. Cien Saude Colet 2017; 22(3):931-939.

10. Varela AR, Schneider BC, Bubach S, Silveira MF, Bertoldi AD, Duarte LSM, Menezes AMB, Domingues MR, Bassani DG. Fetal Neonatal, and post-neonatal mortality in the 2015 Pelotas (Brazil) birth cohort and associated factors. Cad Saude Publica 2019; 35(7):e00072918.

11. Micros PV, Calvo MCM, Colussi CF. Avaliação do desempenho das ações e resultados em saúde da atenção básica. Rev Saude Publica 2017; 51:86.

12. Brasil. Ministério da Saúde (MS). Departamento de Informática do Sistema Único de Saúde. Portal da Saúde. DATASUS. Brasília: MS; 2019.
13. Bisquerra R, Sarriera JC, Martínez F. Introdução à estatística: enfoque informático com o pacote estatístico SPSS. Porto Alegre: Artmed; 2004.

14. Carvalho FCD, Vasconcelos TB, Arruda GMMS, Macena SHM. Modificações nos Indicadores Sociais da região nordeste após a implementação da Atenção Primária. Trab Educ Saude 2019; 17(2):e0018925.

15. Lima JSS, Silva SA. Fuzzy logic application and cluster analysis in the quality of the beverage from conilon coffee. Coffee Sci 2018; 13(4):439-447.

16. Sanders LSC, Pinto FJM, Medeiros CRB, Sampaio RMM, Viana RAA, Lima KJ. Mortalidade infantil: análise de fatores associados em uma capital do Nordeste brasileiro. Cad Saude Colet 2017; 25(1):83-89.

17. Silva ESA, Paes NA. Programa Bolsa Família e a redução da mortalidade infantil nos municípios do Semiárido brasileiro. Cien Saude Colet 2019; 24(2):623-630.

18. Instituto Braseileiro de Geografia e Estatística (IBGE). Malha dos limites politico administrativo 2015. Brasília: IBGE; 2015.

19. Macinko J, Mendonça CS. Estratégia saúde da família, um forte modelo de Atenção Primária à Saúde que traz resultados. Saude Debate 2018; 42(n. esp. 1):1837.

20. Conill EM, Xavier RD, Piola SF, Silva SF, Barros HS, Báscolo E. Determinantes sociais, condicionantes e desempenho dos serviços de saúde em países da América Latina, Portugal e Espanha. Cien Saude Colet 2018; 23(7):2171-2186.

21. Fundação Oswaldo Cruz (Fiocruz). Centro de Estudos Estratégicos Fiocruz. Abrasco alerta para o aumento da mortalidade infantil e materna no Brasil [Internet]. 2018 [acessado 2019 jan 12]. Disponível em: https:// cee.fiocruz.br/?q=Abrasco-alerta-para-o-aumentoda-mortalidade-infantil-e-materna-no-Brasil.

22. Alfradique ME, Bonolo PF, Dourado I, Lima-Costa MF, Macinko J, Mendonça CS, Oliveira VB, Sampaio LFR, Simoni C, Turci MA. Internações por condições sensíveis à atenção primária: a construção da lista brasileira como ferramenta para medir o desempenho do sistema de saúde (Projeto ICSAP - Brasil). Cad Saude Publica 2009; 25(6):1337-1349.

23. Neves RG, Flores T R, Duro SMS, Nunes BP, Tomasi E. Tendência temporal da cobertura da Estratégia Saúde da Família no Brasil, regiões e Unidades da Federação, 2006-2016. Epidemiol Serv Saude 2018; 27(3):e2017170.

24. Instituto Brasileiro de Geografia e Estatística (IBGE). Conheça cidades e estados do Brasil [Internet]. 2017 [acessado 2019 jan 12. Disponível em: https://cidades. ibge.gov.br/cidades 2019.

25. Faria R, Santana P. Variações espaciais e desigualdades regionais no indicador de mortalidade infantil do estado de Minas Gerais, Brasil. Saude Soc 2016; 25(3):736-749.

26. Mario DN, Rigo L, Boclin KLS, Malvestio LMM, Anziliero D, Horta BL, Wehrmeister FC, Martínez-Mesa J. Qualidade do Pré-Natal no Brasil: Pesquisa Nacional de Saúde 2013. Cien Saude Colet 2019; 24(3):12231232 . 
27. Silva G, Alves CRL. Avaliação do grau de implantação dos atributos da atenção primária à saúde como indicador da qualidade da assistência prestada às crianças. Cad Saude Publica 2019; 35(2):e00095418.

Artigo apresentado em 24/04/2020

Aprovado em 05/08/2020

Versão final apresentada em 07/08/2020

Editores-chefes: Romeu Gomes, Antônio Augusto Moura da Silva 